\title{
Adiponectin inhibits cardiac arrest/cardiopulmonary resuscitation-induced apoptosis in brain by increasing autophagy involved in AdipoR1-AMPK signaling
}

\author{
YARONG HE ${ }^{1,2^{*}}$, BOFU LIU ${ }^{1,2 *}$, PENG YAO ${ }^{1,3}$, YUMING SHAO ${ }^{1,3}$, YANWEI CHENG $^{1,3}$, \\ JIE ZHAO ${ }^{1,3}$, JIANG WU ${ }^{3}$, ZHI WEI ZHAO ${ }^{4}$, WEN HUANG ${ }^{5}$, THEODORE A. CHRISTOPHER ${ }^{6}$, \\ BERNARD LOPEZ ${ }^{6}$, XINLIANG MA ${ }^{6}$ and $\mathrm{YU} \mathrm{CAO}^{1,2}$ \\ ${ }^{1}$ Emergency Medicine Department, West China Hospital; ${ }^{2}$ Disaster Medicine Center, \\ ${ }^{3}$ West China Clinical Medical School; ${ }^{4}$ West China School of Preclinical and Forensic Medicine; \\ ${ }^{5}$ Laboratory of Ethnopharmacology, Regenerative Medicine Research Center, West China Hospital, Sichuan University, \\ Chengdu, Sichuan 610041, P.R. China; ${ }^{6}$ Emergency Medicine Department, Thomas Jefferson University Hospital, \\ Philadelphia, PA 19107, USA
}

Received June 7, 2019; Accepted April 4, 2020

DOI: $10.3892 / \mathrm{mmr} .2020 .11181$

\begin{abstract}
Emerging evidence suggests that both apoptosis and autophagy contribute to global cerebral ischemia-reperfusion (GCIR)-induced neuronal death, which results from cardiac arrest (CA). However, the mechanism of how GCIR may affect the balance between apoptosis and autophagy resulting from CA remains to be elucidated. Additionally, the role of adiponectin (APN) in reversing the apoptosis and autophagy induced by GCIR following cardiac arrest-cardiopulmonary resuscitation (CA-CPR) is unclear. Thus, the aim of the present study was to investigate how GCIR affect the apoptosis and autophagy in response to CA and to clarify whether APN may alter the apoptosis and autophagy of neuronal death in GCIR-injured brain post-CA-CPR. Using normal controls (Sham group) and two experimental groups [CA-CPR-induced GCIR injury (PCAS) group and exogenous treatment with adiponectin post-CA-CPR (APN group)], it was demonstrated that both apoptosis and autophagy were observed simultaneously in the brain subjected to GCIR, but apoptosis appeared to be more apparent. Exogenous administration of APN significantly reduced the formation of malondialdehyde, a marker of oxidative stress and increased the expression of superoxide dismutase, an anti-oxidative enzyme, resulting
\end{abstract}

Correspondence to: Professor Yu Cao, Emergency Medicine Department, West China Hospital, Sichuan University, 37 Guoxue Road, Chengdu, Sichuan 610041, P.R. China

E-mail: 1362960346@qq.com.com

*Contributed equally

Key words: adiponectin, cardiopulmonary resuscitation, global cerebral ischemia-reperfusion injury, oxidative stress, AMP-activated protein kinase in the stimulation of autophagy, inhibition of apoptosis and reduced brain tissue injury ( $\mathrm{P}<0.05$ vs. PCAS). APN treatment increased the expression of APN receptor 1 (AdipR1) and the phosphorylation of AMP-activated protein kinase (AMPK; Ser182) in brain tissues. In conclusion, GCIR induced apoptosis and inhibited autophagy, contributing to brain injury in CA-CPR. By contrast, APN reduced the brain injury by reversing the changes of neuronal autophagy and apoptosis induced by GCIR. The possible mechanism might owe to its effects on the activation of AMPK after combining with AdipR1 on neurons, which suggests a novel intervention against GCIR injury in CA-CPR conditions.

\section{Introduction}

Although improved cardio-pulmonary resuscitation (CPR) strategies have increased the return of spontaneous circulation (ROSC) rate in cardiac arrest (CA) patients, both the discharge survival rate and long-term prognosis of these patients remain poor (1). Global cerebral ischemia-reperfusion (GCIR) injury has been considered as one of the most important contributions to poor prognosis (2). However, the exact mechanisms leading to exacerbated post-GCIR remodeling and poor outcomes in ROSC patients are incompletely understood, and effective therapeutic interventions are limited (3).

Emerging evidence suggests that both apoptosis and autophagy contribute to GCIR-induced neuronal death resulting from CA (4). Numerous studies have demonstrated that oxidative stress occurs during focal cerebral ischemiareperfusion promoting protein oxidation, which could impair neuron protein synthesis and metabolism resulting in inevitable neuron apoptosis (5-7). However, definitive proof of the cause-and-effect relationship between oxidative stress and neuron apoptosis following CA-CPR-induced GCIR has remained elusive.

Autophagy is an evolutionarily conserved stress-response mechanism, which often occurs in ischemia-reperfusion 
(IR)-induced oxidative stress (8). Several studies indicated that switching apoptosis to autophagy protects neurons exposed to focal cerebral ischemia or GCIR induced by vertebral artery occlusion $(9,10)$. However, whether the crosstalk between autophagy and apoptosis affects GCIR injury and improves the survival and neurological outcomes of ROSC patients remains to be elucidated, and the mechanisms are unclear (11).

Adiponectin (APN), also known as GBP-28, apM1, AdipoQ and Acrp30, is a novel adipokine that maintains insulin responsiveness, stimulates mitochondrial biogenesis, inhibits inflammatory response and modulates autophagy and apoptosis in different disease models $(12,13)$. A clinical study has revealed that the level of APN is independently associated with mortality and low plasma APN is related to an increased risk of 5-year mortality following the first-ever ischemic stroke in humans (14). The preliminary experiment of the present study demonstrated that APN relieved GCIR by impairing apoptosis in brain tissues of mice suffering from CA-CPR. It has been shown that, APN markedly decreased neuronal injury and infarct size by inhibiting neuronal apoptosis and alleviating oxidative stress in neurons subjected to middle cerebral artery occlusion (MACO)-induced focal cerebral ischemia (FCI) (15). However, few previous studies had clarified the definitive effects of APN on neuronal apoptosis and autophagy following GCIR induced by CA-CPR, and the underlying mechanisms of these effects remain poorly understood.

The present study reported that APN relieved the brain injury via an adiponectin receptor 1 (AdipoR1)/AMP-activated protein kinase (AMPK)-dependent mechanism in a GCIR mice model induced by CA-CPR.

\section{Materials and methods}

Animals and reagents. In total, 60 adult male C57BL/6 (Chengdu Da Shuo Laboratory Animal Co., Ltd., Chengdu, Sichuan) mice, at 8 weeks of age (body weight, 30-35 g), were bred according to guidelines in compliance with the current international laws and policies (16). All animal studies were approved by the Institutional Animal Care and Use Committee at West China Hospital, Sichuan University (no. 2017071A). The mice were housed in a conventional facility under adequate temperature $\left(23^{\circ} \mathrm{C}\right)$ and humidity $(60 \%)$ conditions with a 12-h light/dark cycle and could freely access food and tap water.

Recombinant mouse APN was provided by Professor Xinliang Ma of the Thomas Jefferson University (Philadelphia, USA). Antibodies against cleaved caspase-3 (cat. no. 9661T, Rabbit, 1:1,000), Bcl-2 (cat. no. 3498T, rabbit, 1:1,000), light chain 3 (LC3) II (cat. no. 3868T, rabbit, 1:1,000), Beclin-1 (cat. no. 3495T, rabbit, 1:1,000), AMPK (cat. no. 4182; rabbit; $1: 1,000$ ), anti- $\beta$-actin (cat. no. 3700 ; mouse; $1: 1,000$ ) and p-AMPK (cat. no. 4186, rabbit, 1:1,000) were purchased from Cell Signaling Technology, Inc. APN receptor 1 (rabbit, 1:1,000) was purchased from Santa Cruz Biotechnology, Inc. Malondialdehyde (MDA; cat. no. A003-1) and superoxide dismutase (SOD; cat. no. A001-1-1) detection kits were purchased from Nanjing Jiancheng Bioengineering Institute. A mouse APN/Acrp30 Quantikine ELISA kit (MRP300) was purchased from R\&D Systems, Inc. IgG horseradish peroxidase
(HRP)-conjugated secondary antibodies were purchased from Beyotime Institute of Biotechnology (cat. no. A0208; 1:10,000) or Applygen Technologies, Inc. (1:100,000; cat. no. C1710).

Experimental protocol. Mice were randomized into various treatment groups using random number table: i) Sham group $(n=20)$ was subjected to invasive operation and injected with $0.9 \%$ saline. The mice experience circulatory arrest for $5 \mathrm{~min}$, 40 successful resuscitated mice were randomly divided into the PCAS and APN groups, ii) PCAS group $(n=20)$, which was subjected to cardiac arrest/cardiopulmonary resuscitation (CA/CPR) and injected with $0.9 \%$ saline; and iii) APN group $(n=20)$, which was subjected to CA/CPR and injected with APN $(0.17 \mathrm{mg} / \mathrm{kg})$ as soon as the resuscitation was successful $(15,17,18)$. Every five mice of each group were invasively monitored for an additional of $0.5,3,6$ and $12 \mathrm{~h}$ of reperfusion and were euthanized to harvest the brain tissue and serum for further measurements.

CA/CPR model. CA/CPR was used to induce global cerebral ischemia in vivo. Mice were anesthetized, which was maintained with $2 \%$ isoflurane, and mechanically ventilated to maintain respiration using a rodent ventilator (RWD, Shen Zhen, China). CA/CPR was induced as previously described (19). A cannula (Fine Science) was inserted into the right femoral artery to measure arterial blood pressure (Columbus Instruments). A PE 10 catheter was placed into the right jugular vein for drug administration. An intubation tube was connected to a ventilator (RWD Life Science) and mice were ventilated with a tidal volume of $150 \mu \mathrm{l}$ and a respiratory rate of $100 \mathrm{bpm}$. Following all the preparatory steps, mice were allowed to stabilize for $10 \mathrm{~min}$, during which blood pressure and temperature were recorded. Cardiac arrest was induced by transesophageal alternating current (AC) current stimulation $(7 \mathrm{~V}, 100 \mathrm{~Hz}$ ) for $180 \mathrm{sec}$. A pause in $\mathrm{AC}$ stimulation was then initiated for 1-2 s to observe the change of electrocardiogram (ECG). As soon as the ventricular fibrillation (VF) reverted spontaneously together with an increase of blood pressure, additional 30-sec burst stimulation was performed until either the VF reappeared and persisted, or until asystole or pulseless electrical activity (PEA) occurred. After 2 min with no intervention, cardiopulmonary resuscitation was initiated via an injection of epinephrine (EPI, $0.04 \mathrm{mg} / \mathrm{kg}, 37^{\circ} \mathrm{C}$; Welman Pharmaceutical Co., Ltd.) into the jugular vein catheter followed by manual chest compressions and ventilation with $100 \%$ oxygen with a tidal volume of $4 \mathrm{ml} / \mathrm{kg}$ and a respiratory rate of $100 \mathrm{bpm}$. Manual chest compression at a rate of 200 compressions per minute with equal compression-relaxation duration was always performed by the same investigator who was blinded to the hemodynamic monitor tracings and guided only by audio tones. Compression depth was approximately $1 / 3$ of the anteroposterior chest diameter at maximal compression. ROSC was defined as the return of ventricular rhythm with a mean aortic pressure of $\geq 20 \mathrm{mmHg}$ for a minimum of $5 \mathrm{~min}$ (20). When there was a failure to restore spontaneous circulation after $10 \mathrm{~min}$, resuscitation efforts were discontinued. The flow chart of the experimental protocol is given in Fig. 1.

The procedure described above was similar for the CA/CPR and sham animals, except that the control animals 


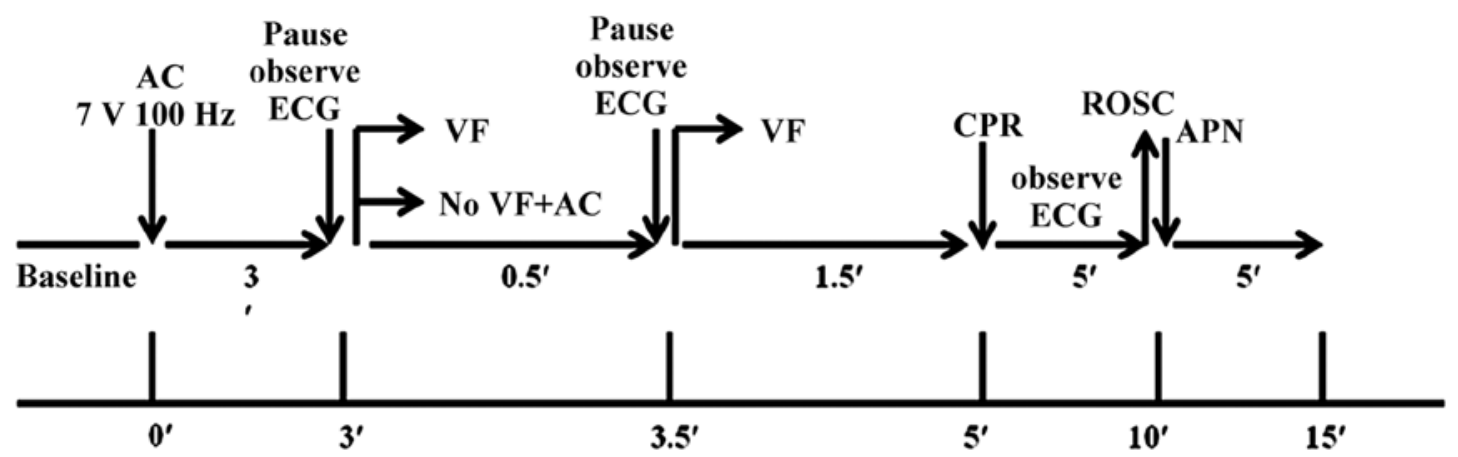

Figure 1. Flow chart of the experimental protocol. The bottom line indicates cumulative elapsed time. The top line indicates elapsed mean intervals until the start of each intervention. AC, alternating current; ECG, electrocardiogram; VF, ventricular fibrillation; CPR, cardiopulmonary resuscitation; ROSC, Return of spontaneous circulation; ', minute.

were injected with isotonic saline instead of epinephrine and did not experience chest compressions.

Tissue processing. The brain tissue was harvested, followed by post-fixing in the $4 \%$ paraformaldehyde overnight at room temperature or homogenizing with lysis buffer (cat. no. P0013B; Beyotime Institute of Biotechnology). The homogenized supernatants and serums were stored in $-80^{\circ} \mathrm{C}$ for western blot analysis and biochemistry indexes.

Histology. Tissue sections were stained with hematoxylin and eosin (H\&E), following standard protocols (21). Briefly, the mice were sacrificed and intracardially perfused with $1 \%$ PBS, followed by $4 \%$ paraformaldehyde overnight at room temperature and were paraffin-embedded. Then, the tissues were cut at the thickness of $5 \mu \mathrm{m}$, and stained using the hematoxylin and eosin (HE) via standard processes at room temperature for 4-10 min. The images were captured using the upright light microscope, according to the previous study described (21).

TUNEL assay. TUNEL staining was used to evaluate the apoptosis of neurons. The brain tissues were fixed with $4 \%$ paraformaldehyde for $12 \mathrm{~h}$ at room temperature, paraffinembedded and cut into 5- $\mu \mathrm{m}$ sections for the TUNEL staining by using the in situ Apoptosis Detection kit (Roche Diagnostics). Cxylene was used to de-paraffinize the paraffin-embedded brain tissues for $20 \mathrm{~min}$, and ethanol $(75,85,95$ and $100 \%$ for $3 \mathrm{~min}$ ) series were used to rehydrate the brain tissues. The brain tissues were incubated with the proteinase $\mathrm{K}$ (at final concentration of $20 \mu \mathrm{g} / \mathrm{ml}$ in $10 \mathrm{mM}$ Tris $/ \mathrm{HCl}$ ) at $37^{\circ} \mathrm{C}$ for $30 \mathrm{~min}$. The endogenous peroxidase activity was blocked with $0.3 \%$ $\mathrm{H}_{2} \mathrm{O}_{2}$ in methanol for $10 \mathrm{~min}$ at room temperature. The cerebral cortex slices were permeabilized by using $0.1 \%$ sodium citrate and $0.1 \%$ Triton-X-100 for $5 \mathrm{~min}$. Then, the slices were washed three times with PBS for $10 \mathrm{~min}$, and were incubated using TUNEL reaction mixture at $37^{\circ} \mathrm{C}$ for $60 \mathrm{~min}$. The slices were incubated using a convertor-POD in humidity chamber for $30 \mathrm{~min}$ at $37^{\circ} \mathrm{C}$. The slices were washed with PBS again for three times, and the color was developed by using a DAB substrate solution for $15 \mathrm{~min}$ at room temperature. Finally, the slices were observed using the light microscopy, and cells with an apoptotic morphology and TUNEL-staining positive cells were identified as apoptotic cells $(21,22)$.
Immunofluorescence staining. Immunofluorescence staining was performed according to a previous study (23). Briefly, paraffin sections made in the preparation of HE staining, were deparaffinized, rehydrated and pretreated with an antigen unmasking solution (Vector Laboratories, Inc.) for $8 \mathrm{~min}$ followed by blocking with peroxidase blocking reagent (Dako; Agilent Technologies, Inc.) and 3\% goat serum (ABC-Elite kit; Vector Laboratories, Inc.). The sections were then incubated overnight with the polyclonal goat anti-rabbit cleaved-caspase 3 and LC3II antibody at $4^{\circ} \mathrm{C}$, followed by a biotinylated anti-goat secondary antibody $(1: 100,000$; cat. no. C1711; Applygen Technologies, Inc.) at $4^{\circ} \mathrm{C}$ for $30 \mathrm{~min}$. The secondary antibody solution was discarded and DAPI solution $(1 \mu \mathrm{g} / \mathrm{ml})$ was applied directly on slides for $5 \mathrm{~min}$ at room temperature. After washing slides three times each for $10 \mathrm{~min}$ in $0.05 \%$ TBS-Tween 20, the slides were incubated with filtered Sudan Black B solution directly for $30 \mathrm{sec}$ in the dark at room temperature. A small amount of fluorescent mounting medium (50-100 $\mu$ i; cat. no. BL701A; Biosharp) and a cover slip was placed over the specimen, avoiding bubbles. Then, clear nail polish was used to seal the sides of the cover glass to the slide. A negative control section was always included in which the primary antibody was substituted by the corresponding isotype control.

Western blot analysis. Brain tissue was homogenized with lysis buffer. The homogenates were centrifuged at $1,000 \mathrm{xg}$ for $10 \mathrm{~min}$ at $4^{\circ} \mathrm{C}$. The protein concentration of the supernatant was measured using the Bradford method (5000001, Bio-Rad Laboratories, Inc.). Equal amounts of proteins at $20 \mu \mathrm{g}$ derived from each sample were separated on $10 \%$ SDS-PAGE gels (Thermo Fisher Scientific, NPO335BOX) and electroblotted onto polyvinylidene fluoride membranes (EMD Millipore, IPVH00010). After blocking by 5\% milk for $30 \mathrm{~min}$ at room temperature, membranes were incubated overnight at $4^{\circ} \mathrm{C}$ with antibodies against target proteins. The membranes were probed with HRP-conjugated secondary antibodies at room temperature for $1 \mathrm{~h}$. Immunoreactive protein bands were visualized by using an enhanced chemiluminescence detection system (GE Healthcare Life Sciences) according to the manufacturer's protocol. Densitometry data were analyzed with ImageJ 1. 8. 0 (National Institutes of Health). 
Table I. Comparison of vital signs in each group (means \pm standard errors of the mean).

\begin{tabular}{lcccc}
\hline Vital sign & Sham & PCAS group & APN group & P-value \\
\hline HR $(\mathrm{bpm})$ & $444.60 \pm 33.37$ & $466.0 \pm 37.34$ & $471.8 \pm 18.15$ & 0.36 \\
MAP $(\mathrm{mmHg})$ & $83.00 \pm 5.09$ & $82.40 \pm 3.91$ & $82.26 \pm 4.44$ & 0.28 \\
\hline
\end{tabular}

One way analysis of variance followed by the Tukey post hoc test for pairwise comparisons were used to compare the difference between the 3 groups. PCAS, post cardiac arrest syndrome; APN, adiponectin; HR, heart rate; MAP, mean arterial pressure.

Table II. Comparison of HR and MAP for the three group after 30-min reperfusion (means \pm standard errors of the mean).

\begin{tabular}{lcccc}
\hline Vital sign & Sham & PCAS group & APN group & P-value \\
\hline HR $(\mathrm{bpm})$ & $489.60 \pm 25.17$ & $466.50 \pm 33.34$ & $473.80 \pm 18.15$ & 0.48 \\
MAP $(\mathrm{mmHg})$ & $88.00 \pm 3.09$ & $70.40 \pm 5.91$ & $73.26 \pm 3.44$ & 0.33 \\
\hline
\end{tabular}

One way analysis of variance followed by the Tukey post hoc test for pairwise comparisons were used to compare the difference between the 3 groups. PCAS, post cardiac arrest syndrome; APN, adiponectin; HR, heart rate; MAP, mean arterial pressure.

SOD and MDA analyses. The tissue level of MDA and SOD contents was analyzed following the manufacturer's instructions (Nanjing Jiancheng Bioengineering Institute), as previously described $(24,25)$. The bicinchoninic acid method was used to test tissue protein concentration. The content of MDA was calculated as $\mathrm{nmol} / \mathrm{mg}$ protein and the activity of SOD was expressed as U/mg protein.

ELISA analysis. The plasma level of APN was determined by using an ELISA kit (MRP300) from R\&D Systems, Inc. according to the manufacturer's instructions.

Statistical analysis. Data are presented as the means \pm standard errors of the mean. All statistical analyses were performed using IBM SPSS Statistics 19.0 (IBM Corp.). Differences of the means among the groups were statistically analyzed with one-way or two-way analysis of variance followed by the Tukey post hoc test for pairwise comparisons (details in the Figure legends). For all statistical tests, $\mathrm{P}<0.05$ was considered to indicate a statistically significant difference.

\section{Results}

Physiological parameters. The HRs of the groups i, ii and iii pre-CA were $444.60 \pm 33.37,466.0 \pm 37.34$ and $471.8 \pm 18.15 \mathrm{bpm}$, respectively ( $\mathrm{P}>0.05)$. MAPs of the 3 groups were 83.00 \pm 5.09 , $82.40 \pm 3.91$ and $82.26 \pm 4.44 \mathrm{mmHg}$, respectively $(\mathrm{P}>0.05$; Table I). In addition, the chest compression manner affected the HRs and MAPs without significant differences among the 3 groups at 30 min after ROSC (Table II).

APN relieves GCIRI. H\&E staining of the cerebral cortex indicated that the PCAS and APN groups appeared to experience neuronal death at $0.5 \mathrm{~h}$ of reperfusion and neuronal activity apoptosis occurred at 3 and $6 \mathrm{~h}$ of reperfusion. Parts of the blood vessels and the parenchyma mesh shape changed (microscopic liquefactive necrosis) in the PCAS group, whereas the severity was significantly reduced in the APN group (Fig. 2).

APN inhibits the GCIR-induced apoptosis in brain tissues and modulated the activation of AMPK induced by GCIR. To further determine whether GCIR induces apoptosis in the brain tissues, DAB-mediated TUNEL and immunofluorescence staining analysis were performed. The results demonstrated that APN relieved the apoptosis after 0.5 to $12 \mathrm{~h}$ of reperfusion (Fig. 3A and B).

GCIR significantly induced the expression of apoptotic protein cleaved caspase- 3 in the brain tissues at all time points following reperfusion in the PCAS group compared to the Sham group $(\mathrm{P}<0.05)$. Conversely, APN post-treatment significantly inhibited the induction of cleaved caspase-3 expression by GCIR and nearly reversed it to the normal level $(\mathrm{P}<0.05$; Fig. 3C and D).

The anti-apoptotic protein, Bcl-2, increased instantly following the initiation of GCIR, which seemed to be significantly higher than the Sham group at 0.5 and $3 \mathrm{~h}$ of reperfusion (Fig. 3E). APN post-treatment increased the expression of Bcl-2 induced by GCIR from 3 to $12 \mathrm{~h}$ ( $\mathrm{P}<0.05$; Fig. $3 \mathrm{C}$ and $\mathrm{E})$.

Chen et al (26) demonstrated that UVB radiation impairs the autophagy response via the phosphorylation of AMPK at the site of Ser182, as well as stimulating reactive oxidative stress in keratinocytes. To determine the effects of GCIR on AMPK activation in brain tissue, the p-AMPK (Ser182)/AMPK ratio was measured. The results indicated that GCIR significantly activated AMPK signaling with a significant elevation of p-AMPK (Ser182)/AMPK ratio at early as $0.5 \mathrm{~h}$ of reperfusion $(\mathrm{P}<0.05)$. However, this effect was later inhibited, especially at 6 and $12 \mathrm{~h}$ of reperfusion $(\mathrm{P}>0.05)$. In contrast, APN post-treatment activated AMPK signaling from $3 \mathrm{~h}$ of reperfusion $(\mathrm{P}<0.05$; Fig. $3 \mathrm{C}$ and $\mathrm{F})$.

APN irrigates GCIR-induced autophagy in brain tissues. LC3II was significantly increased in the GCIR-induced brain 


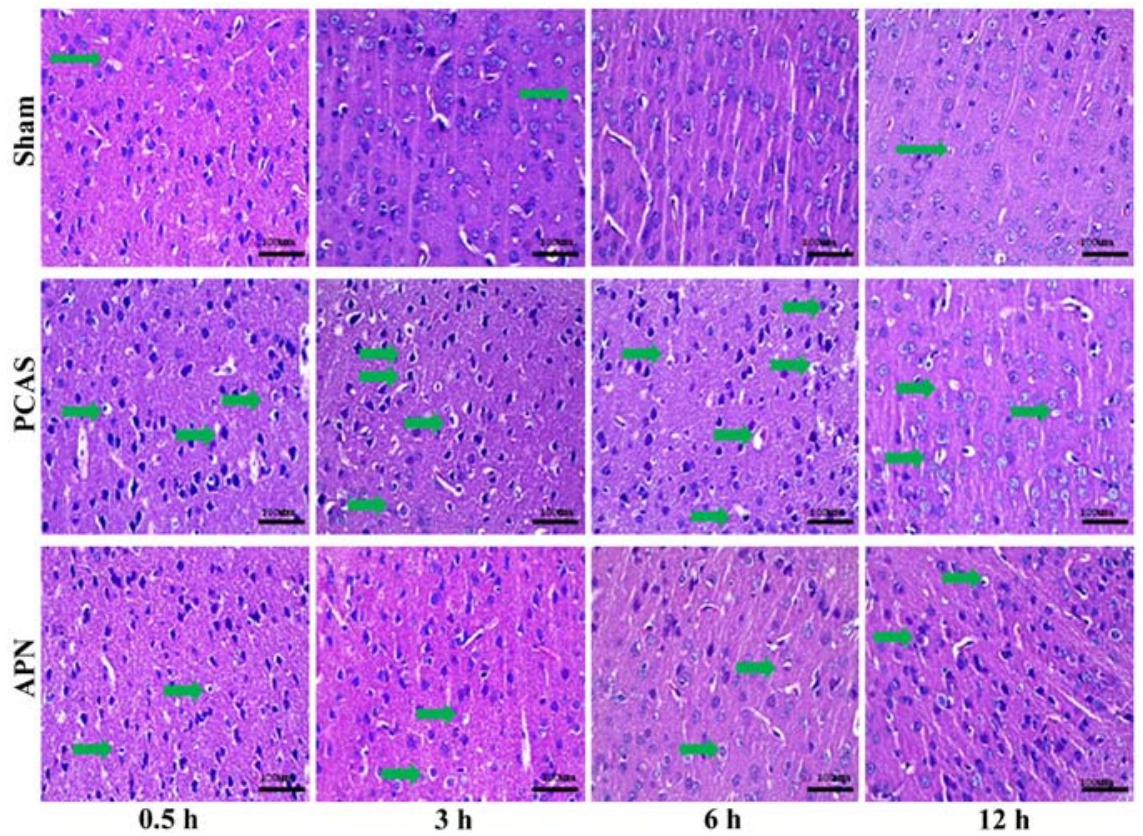

Figure 2. The pathological change of different groups. PCAS and APN group exhibited neuron apoptosis at $0.5 \mathrm{~h}$ of reperfusion and neuron cavities occurred at 3 and $6 \mathrm{~h}$ of reperfusion (arrows indicated the apoptotic or necrosis cell). Scale bar, $100 \mu \mathrm{m}$. PCAS, post cardiac arrest syndrome; APN, adiponectin.

A

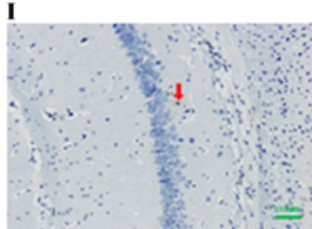

B

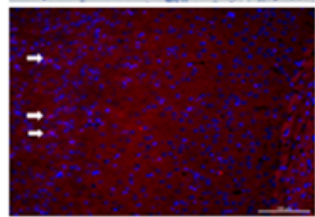

D

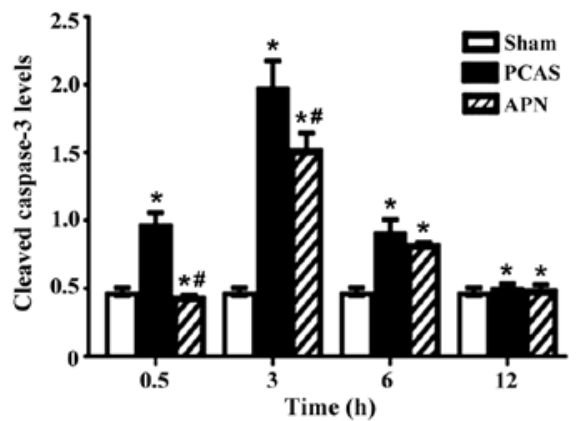

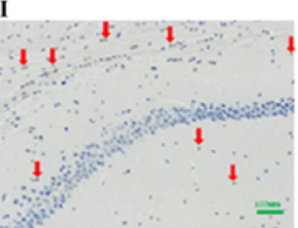

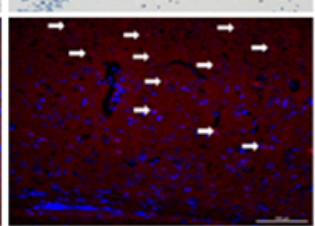

$\mathbf{E}$
III
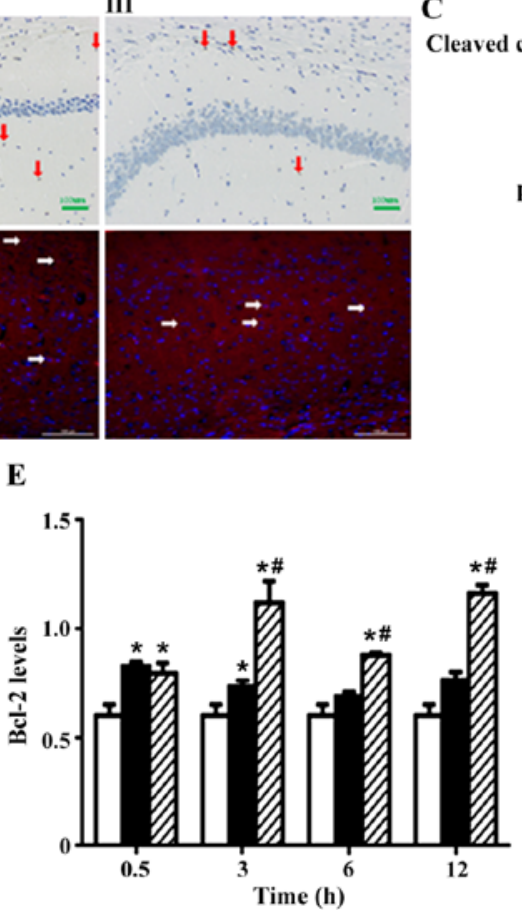

C

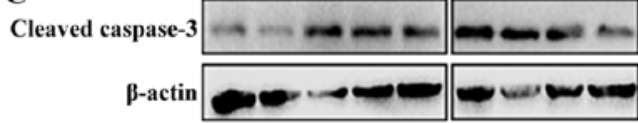

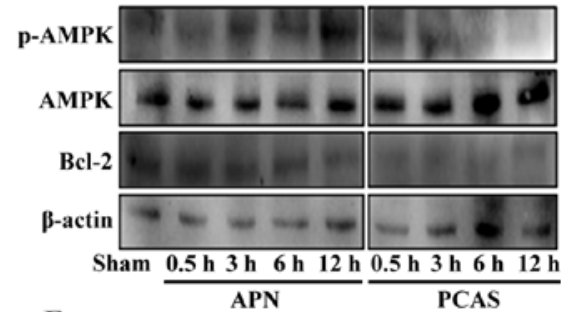

F

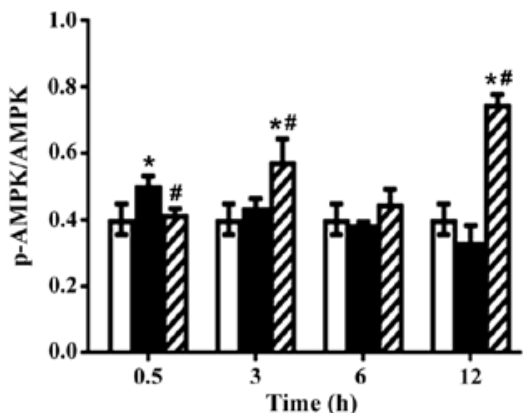

Figure 3. Adiponectin inhibited GCIR-induced apoptosis in brain tissues. (A) TUNEL-positive cells were detected by HRP-conjugated probes with DAB staining in brain tissues of different groups; I: Sham group, II: PCAS group, III: APN group, arrows indicate apoptotic cells. (B) Expression of cleaved casepase- 3 in the cortex of different groups mice at $3 \mathrm{~h}$ of reperfusion; I: Sham group, II: PCAS group, III: APN group, arrows indicated the apoptotic cells. Nuclei were stained by DAPI (blue). Apoptotic protein cleaved casepase-3, anti-apoptotic protein Bcl-2 and activation of AMPK were measured by western blotting in brain tissues of different groups. (C) The grayscale values of (D) casepase-3, (E) Bcl-2 and (F) activation of AMPK in different groups. Data are presented as means \pm standard errors of the mean ( $\mathrm{n}=5$ per group). ${ }^{*} \mathrm{P}<0.05$ vs. Sham group. ${ }^{*} \mathrm{P}<0.05$ vs. PCAS group. GCIR, global cerebral ischemia-reperfusion; HRP, horseradish peroxidase; DAB, 3,3'-diaminobenzidine; DAPI, 4',6-diamidino-2-phenylindole; AMPK, AMP-activated protein kinase; APN, adiponectin; PCAS, post cardiac arrest syndrome.

tissues with or without APN treatment. APN post-treatment increased the LC3II expression in the GCIR-induced brain tissues, with the most significant increase occurring at $3 \mathrm{~h}$ of reperfusion $(\mathrm{P}<0.05$; Fig. $4 \mathrm{~A}$ and $\mathrm{B})$.
In addition, GCIR increased the Beclin- 1 after $0.5 \mathrm{~h}$ of reperfusion, but inhibited it after $3 \mathrm{~h}$ of reperfusion $(\mathrm{P}<0.05)$, with no more significant effects on Beclin-1 in the following time points of reperfusion. By contrast, administration of APN 
A
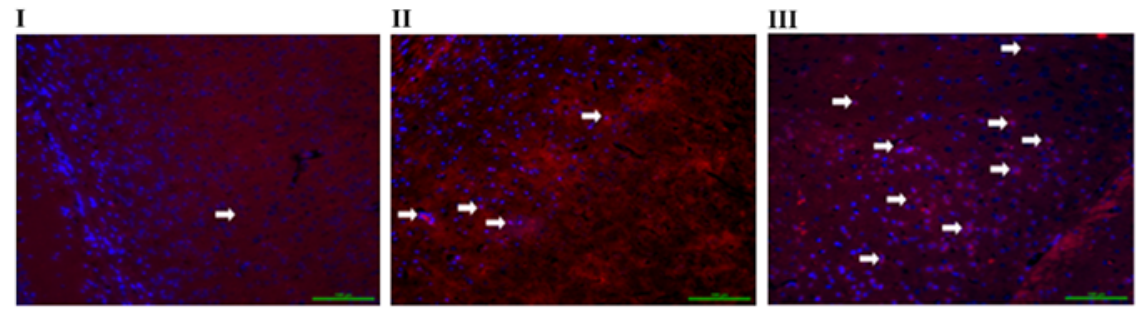

B

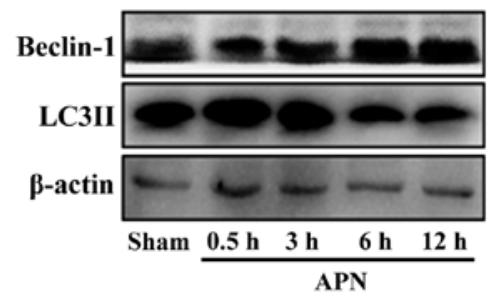

C

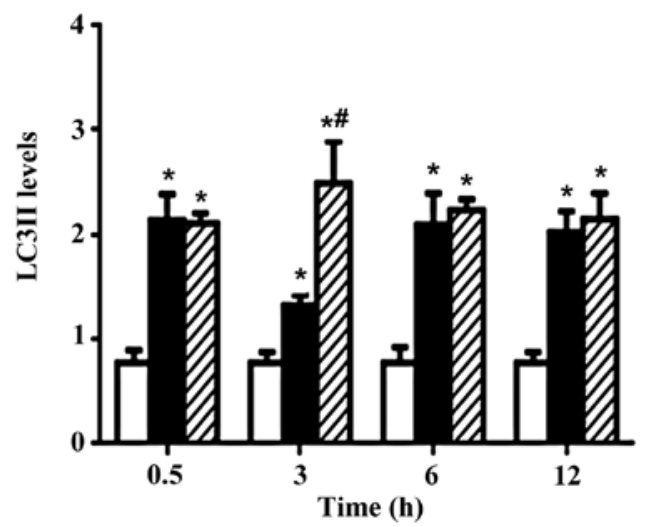

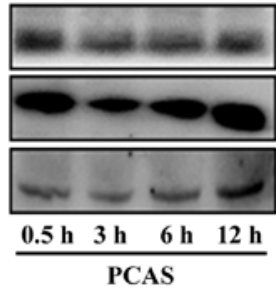

D

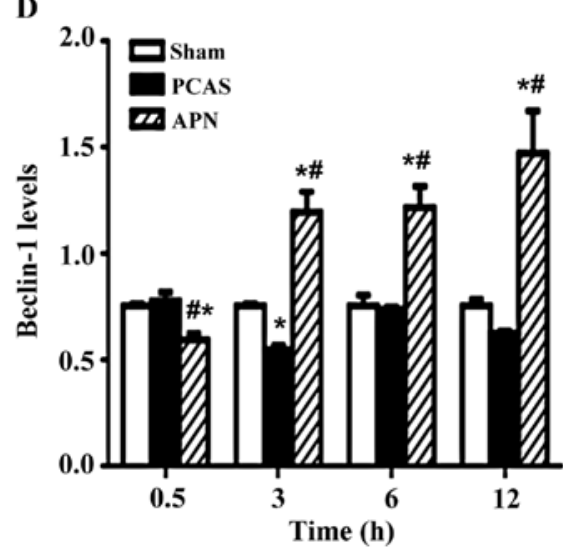

Figure 4. Adiponectin irrigated GCIR-induced autophagy in brain tissues. LC3 II-positive cells were detected by immunofluorescence staining in the cortex of different groups of mice at $3 \mathrm{~h}$ of reperfusion (A) I: Sham group, II: PCAS group, III: APN group, arrows indicated the autophagic cells. Nuclei were stained by DAPI (blue). (B) Effects of adiponectin on GCIR-induced (C) LC3II and (D) Beclin-1 expression levels in brain tissues. Data are presented as means \pm standard errors of the mean ( $\mathrm{n}=5$ per group). ${ }^{\prime} \mathrm{P}<0.05$ vs. Sham group, ${ }^{,} \mathrm{P}<0.05$ vs. PCAS group. GCIR, global cerebral ischemia-reperfusion; LC3, light chain 3; PCAS, post cardiac arrest syndrome; APN, adiponectin.

reversed the effect of GCIR on Beclin-1, except for the $0.5 \mathrm{~h}$ of reperfusion (Fig. 4C and D).

APN alleviates the GCIR-induced unbalance of oxidative and anti-oxidative stress in brain tissues. GCIR significantly induced the formation of MDA in brain tissues after 0.5, 6 and $12 \mathrm{~h}$ of reperfusion $(\mathrm{P}<0.05)$, which was significantly decreased by the post-treatment of APN at $0.5,6$ and $12 \mathrm{~h}$ of reperfusion $(\mathrm{P}<0.05$; Fig. 5A).

The anti-oxidative SOD was stimulated by GCIR at all time points of reperfusion $(\mathrm{P}<0.05)$, peaking at $3 \mathrm{~h}$ of reperfusion. Post-treatment of APN further enhanced the stimulation of GCIR on SOD formation as early as $0.5 \mathrm{~h}$ of reperfusion $(\mathrm{P}<0.05)$, peaking at $3 \mathrm{~h}$ of reperfusion $(\mathrm{P}<0.05$; Fig. $5 \mathrm{~B})$.

Variation of serum APN level and its receptor expressed in different groups. GCIR significantly increased the serum APN level following 3 and $6 \mathrm{~h}$ of reperfusion $(\mathrm{P}<0.05)$, peaking at $3 \mathrm{~h}$. APN post-treatment accelerated the increase of serum APN level induced by GCIR, with its peak concentration appearing as early as $0.5 \mathrm{~h}$ of reperfusion $(\mathrm{P}<0.05)$. However, this effect gradually disappeared, because the serum APN level decreased to less than that of the PCAS group from 3 to $12 \mathrm{~h}$ of reperfusion (Fig. 6A).
GCIR dramatically influenced the AdipoR1 expression at different ROSC time points. For example, GCIR slightly increased its expression at $0.5 \mathrm{~h}$ of reperfusion $(\mathrm{P}>0.05)$ and then was it significantly inhibited at $3 \mathrm{~h}$ of reperfusion $(\mathrm{P}<0.05)$. However, it significantly increased at $6 \mathrm{~h}$ of reperfusion, with levels exceeding those of the Sham group. The injected exogenous APN inhibited the AdipoR1 at the early stage of reperfusion $(0.5 \mathrm{~h} ; \mathrm{P}<0.05)$. However, this effect gradually disappeared since the AdipoR1 expression was significantly higher than that in the PCAS group at 3 and $12 \mathrm{~h}$ of reperfusion $(\mathrm{P}<0.05$; Fig. $6 \mathrm{~B})$.

\section{Discussion}

Numerous studies have demonstrated that apoptosis was one of the cell death types in different nervous disease models, such as subarachnoid hemorrhage (27), stroke (28) and craniocerebral trauma (29). Our previous findings showed that neuronal apoptosis also occurred in mice with CA (30). The results of the present study also demonstrated that the activation of the apoptotic protein caspase 3 and expression of the anti-apoptotic protein Bcl-2 in the PCAS group were increased at $0.5 \mathrm{~h}$ after ROSC. These results confirmed that apoptosis 

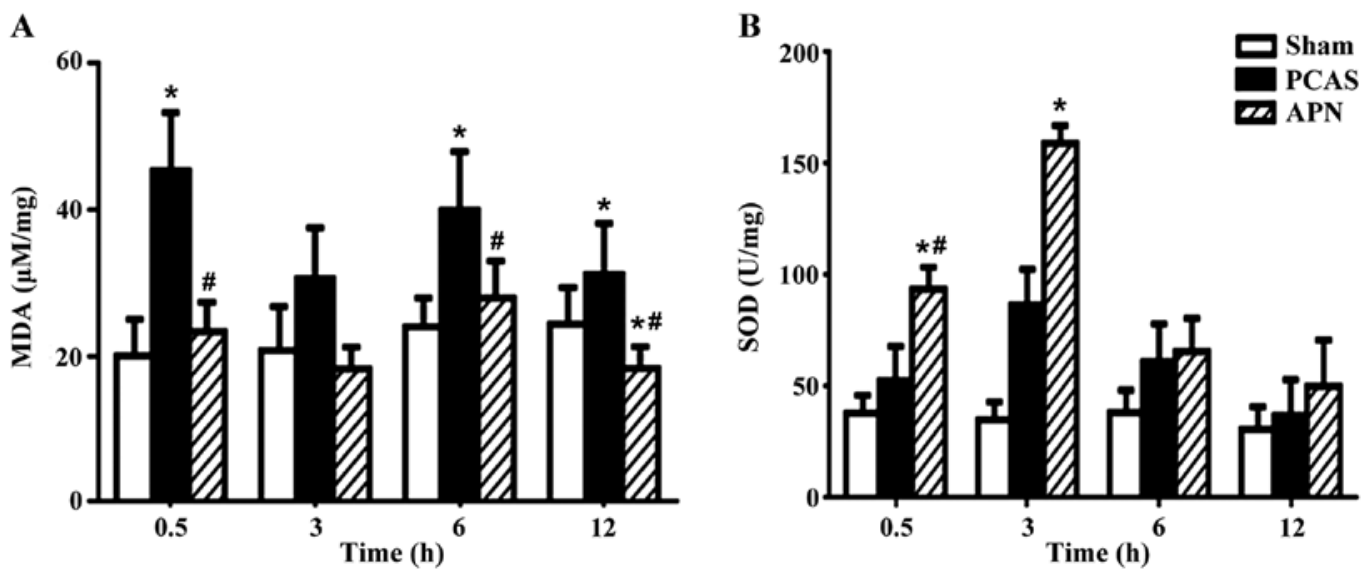

Figure 5. Adiponectin alleviated the GCIR-induce unbalance of oxidative and anti-oxidative stress in brain tissues. The effects of adiponectin in alleviating the GCIR-induce unbalance of (A) MDA and (B) SOD levels in brain tissues. Data are presented as means \pm standard errors of the mean ( $\mathrm{n}=5$ per group). ${ }^{*} \mathrm{P}<0.05$ vs. Sham group, ${ }^{\#} \mathrm{P}<0.05$ vs. PCAS group. GCIR, global cerebral ischemia-reperfusion; MDA, malondialdehyde; SOD, superoxide dismutase; PCAS, post cardiac arrest syndrome.
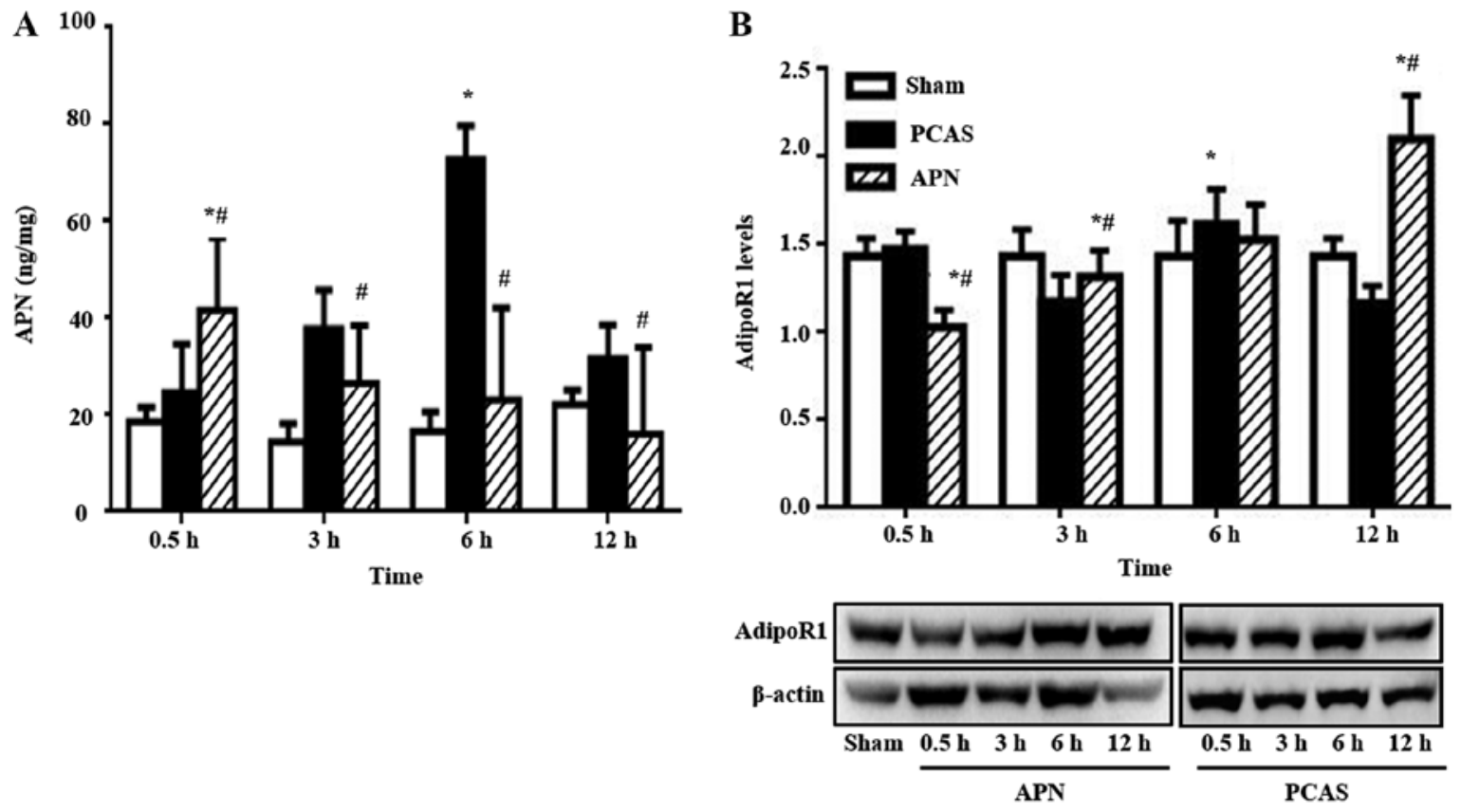

Figure 6. The variation of serum APN level and its receptor expressed in different groups. The variation of (A) serum APN level and (B) its receptor expressed at $0.5,3,6$ and $12 \mathrm{~h}$ of reperfusion in different groups. Representative immunoblots are shown below the quantified data. Data are presented as means \pm standard errors of the mean ( $\mathrm{n}=5$ per group). ${ }^{*} \mathrm{P}<0.05$ vs. Sham group, ${ }^{\sharp} \mathrm{P}<0.05$ vs. PCAS group. APN, adiponectin; PCAS, post cardiac arrest syndrome.

is one of the main mechanisms in the mice brain following GCIR injury induced by CA-CPR.

APN, an adipocyte-derived secretory protein, plays an important role in metabolic (31), anti-inflammatory (32) and anti-oxidative activities (33). A number of studies have reported that APN also specialized in alleviating myocardial, renal and pancreatic ischemic reperfusion injury by reducing apoptosis (34-36). Our previous study indicated that APN reduced the number of apoptotic activities from neurons following GCIR induced by CA-CPR (30). The present study indicated that APN inhibited the apoptosis by reducing the expression of cleaved caspase- 3 in the mice brain tissue following GCIR induced by CA-CPR and promoted the expression of anti-apoptotic protein $\mathrm{Bcl}-2$. These results confirmed the protective effects of APN on GCIR injury induced by CA-CPR by inhibiting apoptosis.

Physiologically, autophagy is a self-protective mechanism of the cells, as it helps maintain the functions of cell structures, which might be damaged by apoptosis (37). However, excessive autophagy also harms the cells, which also results in tissue injury (38). Previous studies have reported that both autophagy and apoptosis are involved in the pathology of cerebral hemorrhage, ischemic stroke and craniocerebral trauma (26-28). Liu et al (39) indicates that arousing autophagy relieves the brain injury by decreasing the apoptotic and necrotic cells. Data in the present study demonstrated that LC3II, a protein subsequently recruited to the autophagosomal membranes when autophagy occurred, promptly increased in the brain tissue from 0.5 to 
$12 \mathrm{~h}$ after ROSC $(\mathrm{P}<0.05)$. By contrast, Beclin-1, an essential mediator of autophagosome formation and another marker the activation of autophagy slightly increased at $0.5 \mathrm{~h}$ after ROSC, but decreased to less than that of the sham group from 3 to $12 \mathrm{~h}$ following reperfusion. These results indicated that GCIR evoked autophagy. In addition, the different expression trends of LC3II and Beclin-1 may result from the interaction between LC3II and Beclin-1 (35). A previous study has demonstrated that activation of LC3I to LC3II inhibits Beclin-1 dissociation from the Beclin-1/Bcl-2 complex, further reducing the polymerization of phosphatidyl inositol kinase (Vps34/PI3K) and finally resulting in cell autophagy (39). This could be the reason LC3II was detected earlier than Beclin-1 in the present study, a finding that was in accordance with that of Guo et al (40).

The present study indicated that APN increased the expression of LC3II and Beclin-1 in the brain tissue simultaneously with inhibition of caspase- 3 activation. To the best of the authors' knowledge, the present study is the first to demonstrate that APN protected the neuron from GCIR injury-induced excessive apoptosis following CA-CPR by promoting autophagy, although Yue et al (41) reported that APN increases the expression of LC3II and Beclin-1 in the skeletal muscle of diabetic mice to reduce apoptotic cells in a diabetic mouse model. The mechanisms of how APN regulates the crosstalk between apoptosis and autophagy in brain tissues injured by GCIR following CA-CPR remain to be elucidated. However, some studies have demonstrated that APN reduces neuronal apoptosis by reducing reactive oxidative stress in an ischemic stroke model $(15,42,43)$. In the present study, the data demonstrated that the APN group had a significantly reduced MDA content and increased SOD level in the brain tissue injured by GCIR following CA-CPR. These results confirmed that APN reduced apoptosis and enhances autophagy by relieving oxidative stress in the brain tissue injured by GCIR following CA-CPR. The present study demonstrated that APN inhibited the activation of AMPK (Ser182) in the brain tissue at the early post-GCIR period $(0.5 \mathrm{~h})$ and then enhanced it at 3 and $12 \mathrm{~h}$ of reperfusion, accompanied by decreased apoptosis and increased autophagy. Although Chen et al (26) have demonstrated this hypothesis in keratinocytes in cardiac and liver cells, no previous studies have suggested that APN activated AMPK (Ser182) signaling pathway to reduce ROS induced-apoptosis to autophagy in GCIR injury induced by CA-CPR. Thus, it is concluded that APN may be involved in the protection of neurons in the brain tissue of post-ROSC mice by regulating the activation of AMPK (Ser182), although other studies have reported the priority activation site being Thr172 $(44,45)$. To the best of the authors' knowledge, the present study has been the first to reveal that APN promoted neuronal autophagy following CA-CPR by activating AMPK and relieving oxidative stress as a result of reducing apoptosis and reducing the brain tissue injury following CA-CPR. In accordance with a previous study, the expression trend of AdipoR1 in the present study was inverse to the concentration of APN, confirming the phenomenon of decreased ligand resulting in increased receptor expression reactively (46).

There are some limitations in the present study. First, it is a short term study to explore the acute injury following GCIR. Therefore, only histological and molecular assessments were performed and no behavioral assessment was conducted. In addition, APN knockout (APN-KO) mice and AMPK inhibitor were not used; thus, further investigations on the effects of APN on the regulation of the crosstalk between apoptosis and autophagy are necessary.

In conclusion, the current study demonstrated that APN administration shortly following ROSC inhibits GCIR injury by reducing apoptosis and increasing autophagy through the activation of the AdipoR1-AMPK signaling pathway, which is therefore important from a translational point of view.

\section{Acknowledgements}

Not applicable.

\section{Funding}

This study was supported by the National Natural Science Foundation of China (grant nos. 81772037 and 81471836to YC and grant no. 81801883 to YRH), the $1 \bullet 3 \cdot 5$ Project for Disciplines of Excellence, West China Hospital, Sichuan University (grant no. ZYJC18019) to YC and the Chengdu Science and Technology Project (grant no. 2018SZ0390) to HFY.

\section{Availability of data and materials}

All data generated or analyzed during the present study are included in this published article.

\section{Authors' contributions}

YH, BLi and ZWZ made contributions to acquisition of data and article drafting. PY, YS, YCh, JZ and JW contributed to analysis and interpretation. YCa, WH, XM, TAC and BLo made substantial contributions to design of the study and revision. All authors read and approved the final manuscript.

\section{Ethics approval and consent to participate}

All animal studies were approved by the Institutional Animal Care and Use Committee at West China Hospital, Sichuan University (approval no. 2017071A).

\section{Patient consent for publication}

Not applicable.

\section{Competing interests}

The authors declare that they have no competing interests.

\section{References}

1. Jerry N: Cardiac arrest and cardiopulmonary resuscitation. Semin Neurol 37: 005-012, 2017.

2. Wang JW, Qiu YR, Fu Y, Liu J, He ZJ and Huang ZT: Transplantation with hypoxia-preconditioned mesenchymal stem cells suppresses brain injury caused by cardiac arrest-induced global cerebral ischemia in rats: Hypoxic stem cells rescue global cerebral ischemia. J Neurosci Resh 95: 2059-2070, 2017.

3. Sanganalmath SK, Gopal P, Parker JR, Downs RK, Parker JC Jr and Dawn BJ: Global cerebral ischemia due to circulatory arrest: Insights into cellular pathophysiology and diagnostic modalities. Mol Cell Biochem 426: 111-127, 2017. 
4. Fakharnia F, Khodagholi F, Dargahi L and Ahmadiani A Prevention of cyclophilin d-mediated mPTP opening using cyclosporine-A alleviates the elevation of necroptosis, autophagy and apoptosis-related markers following global cerebral ischemia-reperfusion. J Mol Neurosci 61: 52-60, 2017

5. Cui D, Shang H, Zhang X, Jiang W and Jia X: Cardiac arrest triggers hippocampal neuronal death through autophagic and apoptotic pathways. Sci Rep 6: 27642, 2016.

6. Mirzaei $\mathrm{H}$ and Regnier F: Protein: Protein aggregation induced by protein oxidation. J Chromatogr B Analyt Technol Biomed Life Sci 873: 8-14, 2008.

7. Liu CL, Ge P, Zhang F and Hu BR: Co-translational protein aggregation after transient cerebral ischemia. Neuroscience 134 1273-1284, 2005.

8. Zhang YP, Zhang Y, Xiao ZB, Zhang YB, Zhang J, Li ZQ and Zhu YB: CFTR prevents neuronal apoptosis following cerebral ischemia reperfusion via regulating mitochondrial oxidative stress. J Mol Med (Berl) 426: 111-127, 2018.

9. Lavandero S, Chiong M, Rothermel BA and Hill JA: Autophagy in cardiovascular biology. J Clin Invest 125: 55-64, 2015.

10. Rami A, Langhagen A and Steiger S: Focal cerebral ischemia induces upregulation of Beclin 1 and autophagy-like cell death Neurobiol Dis 29: 132-141, 2008.

11. Yu B, Ruan M, Liang T, Huang SW, Yu Y, Cheng HB and Shen XC: The synergic effect of tetramethylpyrazine phosphate and borneol for protecting against ischemia injury in cortex and hippocampus regions by modulating apoptosis and autophagy. J Mol Neurosci 63: 70-83, 2017.

12. Ryan F, Khodagholi F, Dargahi L, Minai-Tehrani D and Ahmadiani A: Temporal pattern and crosstalk of necroptosis markers with autophagy and apoptosis associated proteins in ischemic hippocampus. Neurotox Res 34: 79-92, 2018.

13. Berg AH, Combs TP and Scherer PE: ACRP30/adiponectin: An adipokine regulating glucose and lipid metabolism. Trends Endocrinol Metab 13: 84-89, 2002.

14. Efstathiou SP, Tsioulos DI, Tsiakou AG, Gratsias YE, Pefanis AV and Mountokalakis TD: Plasma adiponectin levels and five-year survival after first-ever ischemic stroke. Stroke 36: 1915-1919, 2005.

15. Li X, Guo H, Zhao L, Wang B, Liu H, Yue L, Bai H, Jiang H Gao L, Feng DY and Qu Y: Adiponectin attenuates NADPH oxidase-mediated oxidative stress and neuronal damage induced by cerebral ischemia-reperfusion injury. Biochim Biophys Acta Mol Basis Dis 1863: 3265-3276, 2017.

16. National Research Council (US). Committee Guide for the care and use of laboratory animals. National Academies Press (US), 2011. http://dx. doi. org/10, 17226: 12910.

17. Yao R, Cao Y, He YR, Lau WB, Zeng Z and Liang ZA: Adiponectin attenuates lung fibroblasts activation and pulmonary fibrosis induced by paraquat. PLoS One 10: e0125169, 2015

18. Ding W, Cai Y, Wang W, Ji L, Dong Y, Zhang X, Su M, Liu J, Lu G and Zhang $\mathrm{X}$ : Adiponectin protects the kidney against chronic intermittent hypoxia-induced injury through inhibiting endoplasmic reticulum stress. Sleep Breath 20: 1069-1074, 2016.

19. Chen MH, Liu TW, Xie L, Song FO, He T, Zeng ZY and Mo SR A simpler cardiac arrest model in rats. Am J Emerg Med 25 623-630, 2007

20. Chen MH, Liu TW, Xie L, Song FQ and He T: A comparison of transoesophageal cardiac pacing and epinephrine for cardiopulmonary resuscitation. Am J Emerg Med 24: 545-552, 2006.

21. Song Y, Zhong M and Cai FC: Oxcarbazepine causes neurocyte apoptosis and developing brain damage by triggering Bax/Bcl-2 signaling pathway mediated caspase 3 activation in neonatal rats. Eur Rev Med Pharmacol Sci 22: 250-261, 2018.

22. Bennett MW, O'connell J, O'sullivan GC, Roche D, Brady C, Kelly J, Collins JK and Shanahan F: Expression of Fas ligand by human gastric adenocarcinomas: A potential mechanism of immune escape in stomach cancer. Gut 44: 156-162, 1999.

23. Wolf AM, Wolf D, Avila MA, Moschen AR, Berasain C Enrich B, Rumpold $\mathrm{H}$ and Tilg $\mathrm{H}$ : Up-regulation of the anti-inflammatory adipokine adiponectin in acute liver failure in mice. J Hepatol 44: 537-543, 2006.

24. Zhu X, Tang Z, Cong B, Du J, Wang C, Wang L, Ni X and Lu J: Estrogens increase cystathionine-gamma-lyase expression and decrease inflammation and oxidative stress in the myocardium of ovariectomized rats. Menopause 20: 1084-1091, 2013.

25. Yang T, Mao YF, Liu SQ, Hou J, Cai ZY, Hu JY, Ni X, Deng XM and Zhu XY: Protective effects of the free radical scavenger edaravone on acute pancreatitis-associated lung injury. Eur J Pharmacol 630: 152-157, 2010.
26. Chen X, Li L, Xu S, Bu W, Chen K, Li M and Gu H: Ultraviolet B radiation down-regulates ULK1 and ATG7 expression and impairs the autophagy response in human keratinocytes. J Photochem Photobiol B 178: 152-164, 2018.

27. Han Y, Zhang T, Su J, Zhao Y and Li X: Apigenin attenuates oxidative stress and neuronal apoptosis in early brain injury following subarachnoid hemorrhage. J Clin Neurosci 40: 157-162, 2017.

28. Li Y, Mei Z, Liu S, Wang T, Li H, Li XX, Han S, Yang Y, Li J and $\mathrm{Xu}$ ZD: Galanin protects from caspase-8/12-initiated neuronal apoptosis in the ischemic mouse brain via GalR1. Aging Dis 8: 85-100, 2017.

29. Zhang M, Wu J, Ding H, Wu W and Xiao G: Progesterone provides the pleiotropic neuroprotective effect on traumatic brain injury through the Nrf2/ARE signaling pathway. Neurocrit Care 26: 292-300, 2017.

30. Osellame LD, Blacker TS and Duchen MR: Cellular and molecular mechanisms of mitochondrial function. Best Pract Res Cl En 26: 711-723, 2012

31. Mongardon N, Dumas F, Ricome S, Grimaldi D, Hissem T, Pène F and Cariou A: Postcardiac arrest syndrome: From immediate resuscitation to long-term outcome. Ann Intensive Care 1: 45, 2011.

32. Denicola A and Radi R: Peroxynitrite and drug-dependent toxicity. Toxicology 208: 273-288, 2005.

33. Zhu Y, Yang GY, Ahlemeyer B, Pang L, Che XM, Culmsee C, Klumpp S and Krieglstein J: Transforming growth factor- $\beta 1$ increases bad phosphorylation and protects neurons against damage. Neurosci 22: 3898-3909, 2002

34. Sun Y, Zhao D, Yang Y, Gao C, Zhang X, Ma Z, Jiang S, Zhao L, Chen W, Ren KJ, et al: Adiponectin exerts cardioprotection against ischemia/reperfusion injury partially via calreticulin mediated anti-apoptotic and anti-oxidative actions. Apoptosis 22: 108-117, 2017.

35. Yi W and Yang Q: Adiponectin improves diabetic nephropathy by inhibiting necrotic apoptosis. AMS 15: 1321-1328, 2019.

36. Wang Y, Li Y, Qiao J, Li N and Qiao S: AMPK $\alpha 1$ mediates the protective effect of adiponectin against insulin resistance in INS-1 pancreatic $\beta$ cells. Cell Biochem Funct 37: 625-632, 2019.

37. Wiklund L, Martijn C, Miclescu A, Semenas E, Rubertsson S and Sharma HS: Central nervous tissue damage after hypoxia and reperfusion in conjunction with cardiac arrest and cardiopulmonary resuscitation: Mechanisms of action and possibilities for mitigation. Int Rev Neurobiol 102: 173-187, 2012

38. Xu KY, Zweier JL and Becker LC: Hydroxyl radical inhibits sarcoplasmic reticulum $\mathrm{Ca}(2+)$-ATPase function by direct attack on the ATP binding site. Circ Res 80: 76-81, 1997.

39. Liu Z, Gan L, Wu T, Feng F, Luo D, Gu H, Liu S and Sun C: Adiponectin reduces ER stress-induced apoptosis through PPARa transcriptional regulation of ATF2 in mouse adipose. Cell Death Dis 7: e2487, 2016.

40. Guo F, Jiang T, Song W, Wei H, Wang F, Liu L, Ma L, Yin H Wang Q and Xiong L: Electroacupuncture attenuates cerebral ischemia-reperfusion injury in diabetic mice through adiponectin receptor 1-mediated phosphorylation of GSK-3 3 . Mol Neurobiol 51: 685-695, 2015.

41. Yue L, Zhao L, Liu H, Li X, Wang B, Guo H, Gao L, Feng D and $\mathrm{Qu}$ Y: Adiponectin protects against glutamate-induced excitotoxicity via activating SIRT1-dependent PGC-1 expression in HT22 hippocampal neurons. Oxid Med Cell Longev 2016: 2957354, 2016.

42. Adrie C, Adib-Conquy M, Laurent I, Monchi M, Vinsonneau C, Fitting C, Fraisse F, Dinh-Xuan AT, Carli P, Spaulding C, et al: Successful cardiopulmonary resuscitation after cardiac arrest as a 'sepsis-Like' syndrome. Circulation 106: 562-568, 2002.

43. Gairolla J, Kler R, Modi M and Khurana DJ: Leptin and adiponectin: Pathophysiological role and possible therapeutic target of inflammation in ischemic stroke. Rev Neurosci 28: 295-306, 2017.

44. Duan J, Yin Y, Cui J, Yan J, Zhu Y, Guan Y, Wei G, Weng Y, Wu X, Guo C, et al: Chikusetsu saponin IVa ameliorates cerebral ischemia reperfusion injury in diabetic mice via adiponectin-mediated AMPK/GSK-3beta pathway in vivo and in vitro. Mol Neurobiol 53: $728-743,2016$

45. Piao L, Yu C, Xu W, Inoue A, Shibata R, Li X, Nan Y, Zhao G, Wang H, Meng X, et al: Adiponectin/AdiopR1 signal inactivation contributes to impaired angiogenesis in mice of advanced age. Int J Cardiol 267: 150-155, 2018.

46. Gamberi T, Modesti A, Magherini F, D'Souza DM, Hawke T and Fiaschi T: Activation of autophagy by globular adiponectin is required for muscle differentiation. Biochim Biophys Acta 1863: 694-702, 2016.

This work is licensed under a Creative Commons Attribution-NonCommercial-NoDerivatives 4.0 International (CC BY-NC-ND 4.0) License. 